\title{
Safety assessment and biochemical evaluation of the effect of biogenic silver nanoparticles (using bark extract of C. zeylanicum) on Rattus norvegicus rats
}

\author{
Shukrya H. Alwan ${ }^{1,2,3}$, Muna H. Al-Saeed ${ }^{1}$ and Hussein A. Abid ${ }^{4}$ \\ ${ }^{1}$ Department of Physiology, Pharmacology and Biochemistry, College of Veterinary Medicine, University of Basrah, Basrah, Iraq \\ ${ }^{2}$ Department of Biology, College of Science, University of Baghdad, Baghdad, Iraq \\ ${ }^{3}$ Department of Community Health, Technical Institute of Karbala, AlFurat AlAwsat Technical University, Karbala, Iraq \\ ${ }^{4}$ Department of Medical Laboratory Technology, Technical Institute of Baquba, Middle Technical University, Baqubah, Iraq
}

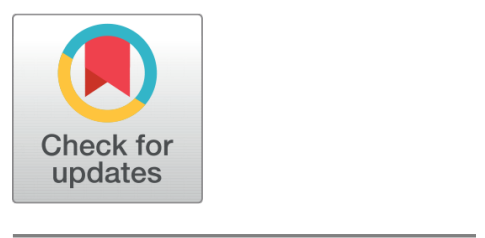

Received 06-07-2021

Revised 24-07-2021

Accepted 25-07-2021

Published 27-07-2021

\section{Corresponding Author}

Shukrya H. Alwan

shuk_hat1129@yahoo.com

Department of Biology, College of Science, University of Baghdad, Baghdad, Iraq

DOI https://doi.org/10.47419/ bjbabs.v2i03.67

\section{Pages: 133-145}

Distributed under the terms of the Creative Commons

Attribution-NonCommercial 4.0 International (CC-BY-NC 4.0), which permits use for any non-commercial purpose, distribution, and reproduction in any medium, provided that the original work is properly cited.

Copyright: (C) 2021 Shukrya H. Alwan, Muna H. Al-Saeed, Hussein A. Abid

\section{ABSTRACT}

Background: Biosynthesized silver nanoparticles (AgNPs) are widely used in various biomedical applications. However, limited reports are currently available about the safety of biofabricated AgNPs using Cinnamomum zeylanicum bark extracts.

Objectives: The current study is aimed to assess the potential toxicity of biosynthesized AgNPs (using C. zeylanicum) by subacute oral administration in experimental rats.

Methods: AgNPs were biofabricated using methanol extract of C. zeylanicum bark and characterized by scanning electron microscopy (SEM) and atomic force microscopy (AFM). Twenty-four Rattus norvegicus female rats were divided into 4 groups ( 6 animals per group) as follows: Group-I was the control, while groups II, III, and IV were given $0.85,1.76$ and $3.53 \mathrm{mg} / \mathrm{kg}$ doses of AgNPs, respectively for 14 consecutive days. After 14 days of oral administration of AgNPs, serum levels of malondialdehyde (MDA), superoxide dismutase (SOD) and catalase (CAT) were measured using an ELISA technique. Serum concentrations of urea, creatinine, aspartate transaminase (AST) and alanine transaminase (ALT) were also determined as well as histopathologic features of the liver and the kidney.

Results: AgNPs did not induce any changes in mean body weight, biochemical parameters (AST, ALT, urea and creatinine), oxidative stress biomarkers (MDA, SOD and CAT) and histopathologic features (of the liver and kidneys) of the treated groups when compared to control group.

Conclusions : Our findings suggest that the oral administration of biogenic AgNPs (biofabricated using methanol extract of C. zeylanicum) to rats at a specific dose is relatively safe and does not show any signs of toxicity.

Keywords cinnamon, green synthesis, histopathology, oxidative stress, silver nanoparticles, subacute toxicity

\section{OPEN ACCESS}




\section{INTRODUCTION}

Over the last few decades, the use of nanoparticles in biomedical applications has increasingly emerged and gained great attention. ${ }^{1,2}$ Similarly, the medicinal activities of many plants are widely investigated and proven. ${ }^{3}$ Among many methods to synthesize nanoparticles, the bio- or green synthesis (i.e. using plants extracts to reduce metal oxides and due to their secondary metabolites) is a widely used method by many researchers. ${ }^{4,5}$ Nanoparticles have unique properties such as small sizes and a large surface to volume ratio, making them ideal for a wide range of applications, particularly in biomedicine. ${ }^{2}$ Although nanoparticles of specific sizes have demonstrated useful mechanical, electrical, and chemical properties, these same desirable properties could also be associated with unwanted bio-toxicological effects. ${ }^{6}$ This raises concerns about the safety of using nanoparticles in biomedical applications.

When compared to metal NPs, in nanomedicine, the nanomaterials synthesized by using natural herbs are preferred because of their biocompatibility, easy stabilization, presence of rich active agents, low cultivation cost, ease of use and safe handling. ${ }^{7}$ Green synthesis employing plant substances is a more secure and environmentally friendly way for producing NPs. Accordingly, plants offer an attractive system for NP synthesis because of their capability to deliver an extensive variety of optional metabolites with a weak potential of toxicity. Also, plant extracts offer a green option for biosynthesis of nanoparticles, such as AgNPs. ${ }^{8,9}$ Cinnamon (Cinnamomum zeylanicum) extracts have additional properties for NPs synthesis because of their phytoconstituents (phenolic and flavonoids), which act as capping and reducing agents for Ag nanoparticles, offering stability and the capacity to regulate the size and form of NPs by adding extra layers. ${ }^{10}$ Gauthami and his colleagues synthesized AgNPs from C. zeylanicum bark extract and utilized it in a medicinal application. ${ }^{10}$ It is expected that the use of plant-based AgNPs in medicine and pharmaceutical interventions will be more widespread, given that these advantages include availability, nontoxicity, multifunctionality, and the diversity of metabolites that help to remove Ag ions from biological systems. ${ }^{11}$

Despite the widespread use of AgNPs from C. zeylanicum methanolic extract in medical applications, there is no scientific evidence of its safety. In order to fill in the gap, this study was aimed at evaluating the safety and toxicity of AgNPs after 14 days of daily oral administration in female Rattus norvegicus rats.

\section{MATERIALS AND METHODS Green synthesis and characterization of AgNPs}

Silver nanoparticles were biofabricated by using methanol extract of $C$. zeylanicum bark

extracts according to the method of Gauthami et al. (2015) with some modifications. ${ }^{10}$ Briefly, $5 \mathrm{~mL}$ of methanol extract of C. zeylanicum bark was added to $200 \mathrm{~mL}$ of $1 \mathrm{mM}$ silver 
nitrate $\left(\mathrm{AgNO}_{3}\right)$. The mixture is then shaken and stored in a dark place for 72 hours. The formation of AgNPs was predicted by color change to dark-brown. Finally, the mixture was centrifuged and the suspension was washed 5 times, repeatedly.

The biosynthesis of AgNPs was monitored periodically and characterized by using scanning electron microscopy (SEM, VEGAIII, Czech Republic) and atomic force microscopy (AFM, Negara, Russian Federation). All chemicals used were of analytical grade and purchased from Sigma-Aldrich (Germany) or from local market (for the cinnamon).

\section{Subacute toxicity study}

Twenty-four Rattus norvegicus healthy female rats (10-12 weeks old, weighing 160-185g) were selected for this study and maintained in the animal house of the College of Veterinary Medicine at the University of Basrah. The rats were housed in standard environmental conditions, fed on a standard pellet diet and water ad libitum. They were divided into four groups $(\mathrm{n}=6)$, as follows: group I, control group (received distilled water only); group II (received $0.85 \mathrm{mg} / \mathrm{kg}$ AgNPs); group III (received $1.76 \mathrm{mg} / \mathrm{kg}$ AgNPs) and group IV (received $3.53 \mathrm{mg} / \mathrm{kg}$ AgNPs) once daily for 14 consecutive days. The body weight of all rats was recorded at day 0 days and at the end of the experiment (day 14). The percentage body weight gain was calculated by using the following equation. ${ }^{12}$

Body weight gain $=\frac{\text { Body weight at the end of the experiment }- \text { Initial body weight }}{\text { Initial body weight }} \times 100 \%$

This subacute toxicity study was designed with slight modification based on earlier procedure of Ebohon et al. (2020). ${ }^{13}$ The rats were left in their cages under observation during the period of study for mortality and any signs of toxicity such as gross behavioral, neurologic, and autonomic toxic signs such as changes in respiration, heart rate, salivation, lacrimation, drowsiness, or convulsions.

The institutional review board and research ethics committee at the College of Veterinary Medicine, University of Basra (Basrah, Iraq) has approved this study (No.: 3/18 on 01/07/2019).

\section{Blood and tissue sample collection}

After the last exposure to AgNPs of C. zeylanicum methanol extract, all animals were euthanized and sacrificed. Blood was collected via heart puncture and allowed to clot for 1 hour and then centrifuged at $3000 \mathrm{rpm}$ for $15 \mathrm{~min}$ to obtain serum. ${ }^{14}$ Biopsies of the liver and kidney, for histological evaluation, were fixed in $10 \%$ formalin. 


\section{Evaluation of oxidative stress, renal and hepatic function}

To assess the potential of AgNPs to induce the oxidative stress, lipid peroxidation biomarker (malondialdehyde, MDA), ${ }^{15}$ and enzymatic antioxidants (superoxide dismutase, SOD and catalase, CAT) ${ }^{16}$ were evaluated in serum samples by the enzyme-linked immunosorbent assay (ELISA) technique using a commercial kit (ElabScience, USA). The test procedure of each biomarker was according to the manufacturer's instructions.

The renal and liver function tests (urea, creatinine, ALT and AST) were measured using kits for Reflotron plus (Roche, Germany), based on the manufactured company's guidelines.

\section{Histopathological features}

After scarifying the animals, at the end of the experiment, some of organs such as kidneys and liver were collected for histopathological examinations. Small biopsy from each animal's organs (liver and kidney) were fixed in $10 \%$ formalin and histopathological processing were performed using hematoxylin and eosin staining. ${ }^{17}$ The sectioned tissues were therefore examined using 10X light microscopy objectives.

\section{Statistical analyses}

GraphPad Prism 8.0 (GraphPad Software, CA, USA) was used to evaluate the data, statistically. Findings was expressed as mean \pm SD. Data was analyzed using one-way analysis of variance (ANOVA) or Kruskal-Wallis, based on the normality test results, followed by post-hoc Tukey's HSD test. Repeated measures were also used to analyzed the difference in body weight before and after AgNPs' administration. The differences were considered statistically significant at a $p$-value of $\leq 0.05$.

\section{RESULTS}

\section{Green synthesis and characterization of AgNPs}

The first step in the fabrication of AgNPs was identified by a distinct change in color from light brown to dark brown, which indicates the formation of AgNPs (Figure 1). Scanning electron microscopy (SEM) and atomic force microscopy (AFM) results showed that the gained AgNPs were smooth, spherical particles, singular or in aggregates with $60-80 \mathrm{~nm}$ sizes (Figures 2 and 3). 


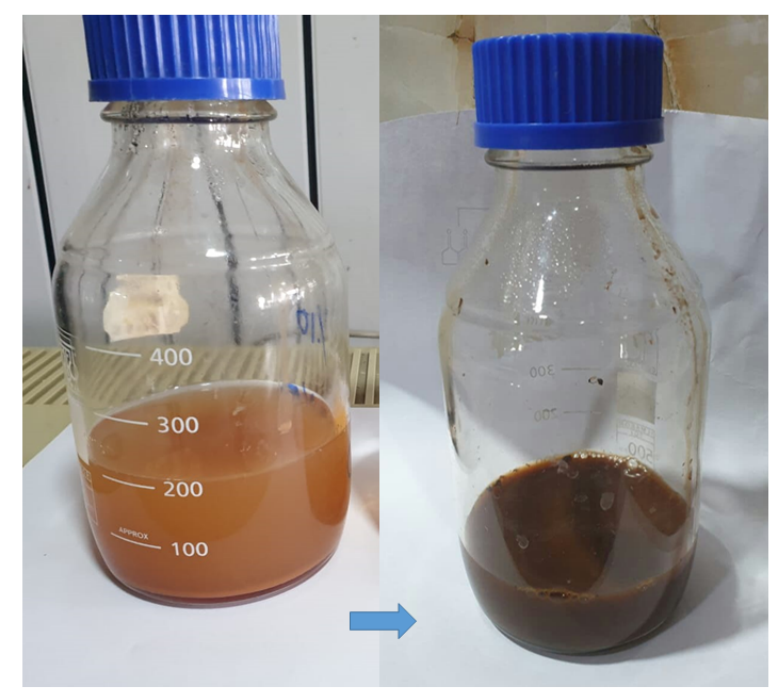

Figure 1 Color change from light brown to dark brown indicates the formation of AgNPs.

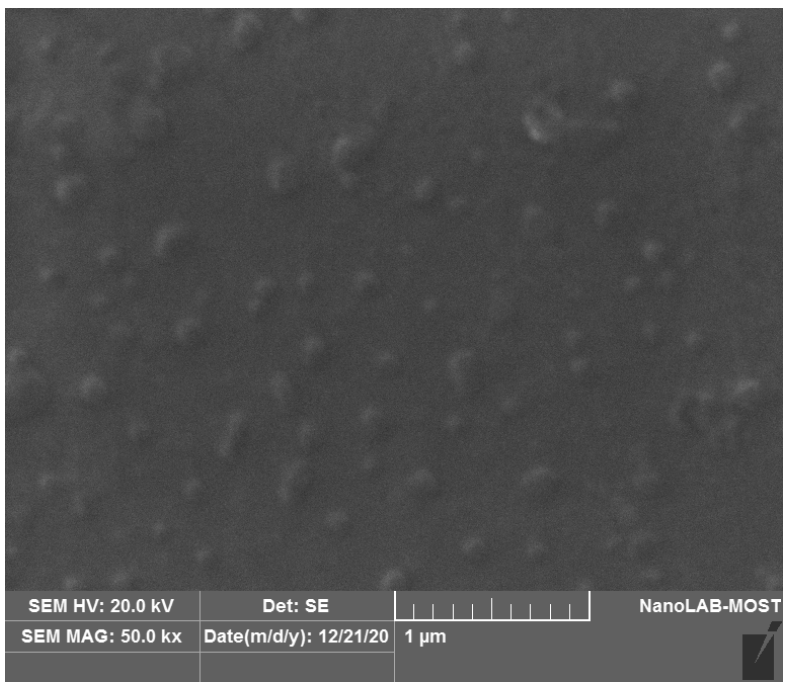

Figure 2 Scanning electron microscopy (SEM) shows the shape and size of AgNPs.

\section{Body weight and toxicity signs}

During the period of the experiment, all rats were observed to detect mortality and any signs of toxicity. In all groups, administration of $0.85,1.76$ and $3.53 \mathrm{mg} / \mathrm{kg}$ of biogenic AgNPs for 14 consecutive days revealed no deaths, so the mortality rate was $0 \%$. In addition, no toxicity signs were observed in any of the groups during the AgNPs administration periods. Table 1 shows the effect of AgNPs on the body weight of rats after 14 days of administration. There were no significant changes $(p>0.05)$ in the body weight of the rats 


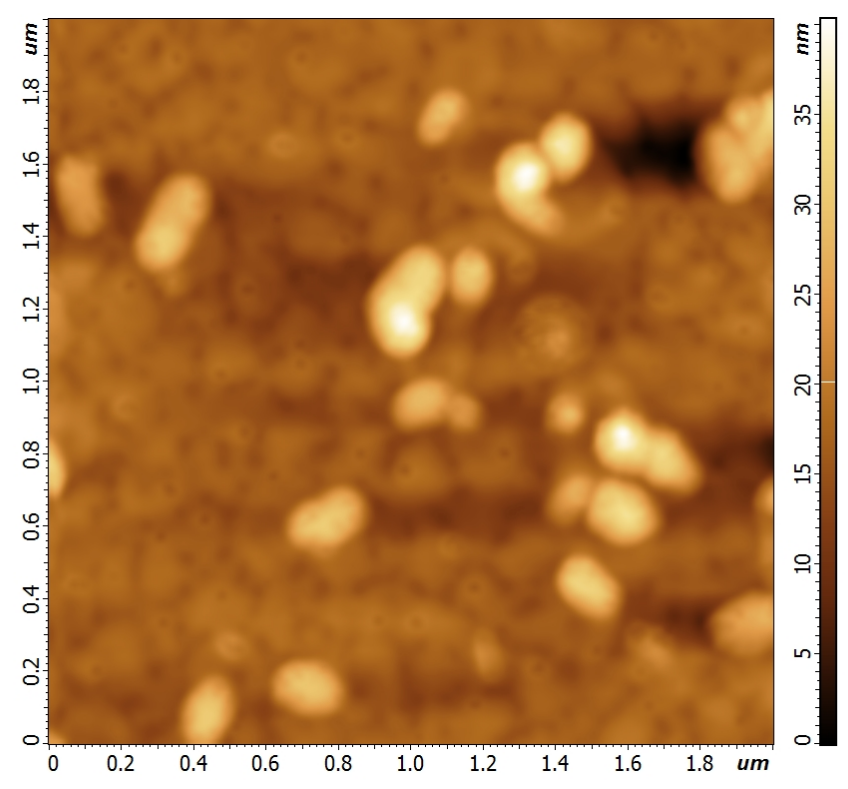

Figure 3 Atomic force microscopy (AFM) image shows two dimensions of AgNPs.

throughout the 14 days of the study in all groups that administration of AgNPs in comparison with the control.

\begin{tabular}{llll}
\hline \multicolumn{2}{l}{ Table $\mathbf{1}$ The effect of AgNPs on body weight $(\mathrm{g})$. } \\
\hline Groups & Day 0 & Day $\mathbf{1 4}$ & Weight gain (\%) \\
\hline I & $173.3 \pm 8.4$ & $181.2 \pm 8.3$ & $7.8 \pm 1.4$ \\
II & $168.7 \pm 6.5$ & $176.3 \pm 6.8$ & $7.6 \pm 2.4$ \\
III & $167.5 \pm 10.3$ & $174.3 \pm 9.8$ & $7.5 \pm 2.9$ \\
IV & $171.5 \pm 10.1$ & $178.0 \pm 10.3$ & $6.5 \pm 1.3$ \\
$\boldsymbol{P}$ & 0.67 & 0.60 & 0.44 \\
\hline
\end{tabular}

Values are mean $\pm S D$, no significant differences at $(p \leq 0.05)$ level . I) control group, II) group received $0.85 \mathrm{mg} / \mathrm{kg}$ AgNPs, III) group received $1.76 \mathrm{mg} / \mathrm{kg}$ AgNPs, IV) group received $3.53 \mathrm{mg} / \mathrm{kg}$ AgNPs.

\section{Oxidative stress, renal and hepatic function}

The effect of oral administration of AgNPs on renal function (serum creatinine and urea levels) is shown in Table 2 . In this context, there were no significant changes $(p>0.05)$ in creatinine and urea levels among the groups when compared with the control group following oral administration of AgNPs. In addition, no significant changes $(p>0.05)$ in serum levels of AST and ALT were observed between groups that received AgNPs compared to the control group (Table 2) after 14-days of NPs' administration. 
Table 2 The effect of AgNPs on renal and liver functions of experimental rats.

\begin{tabular}{lll} 
Groups & Renal function tests & \\
& Urea $(m g / d L)$ & Creatinine $(m g / d L)$ \\
I & $45.00 \pm 7.9$ & $0.24 \pm 0.08$ \\
II & $50.80 \pm 1.9$ & $0.34 \pm 0.11$ \\
III & $46.80 \pm 5.4$ & $0.34 \pm 0.11$ \\
IV & $40.20 \pm 6.9$ & $0.30 \pm 0.07$ \\
P & 0.08 & 0.36 \\
Groups & Liver enzymes & \\
& $A S T(U / L)$ & $A L T(U / L)$ \\
I & $89.60 \pm 8.0$ & $36.17 \pm 4.7$ \\
II & $93.60 \pm 8.4$ & $39.17 \pm 5.8$ \\
III & $101.6 \pm 4.1$ & $44.83 \pm 6.4$ \\
IV & $97.80 \pm 6.5$ & $42.00 \pm 6.8$ \\
$\boldsymbol{P}$ & 0.07 & 0.11 \\
\hline
\end{tabular}

Values are mean $\pm S D$, no significant differences at $(p \leq 0.05)$ level. I) control group, II) group received $0.85 \mathrm{mg} / \mathrm{kg}$ AgNPs, III) group received $1.76 \mathrm{mg} / \mathrm{kg}$ AgNPs, IV) group received $3.53 \mathrm{mg} / \mathrm{kg}$ AgNPs.

\section{Malondialdehyde and enzymatic antioxidants}

There were no significant changes $(p>0.05)$ in the serum levels of oxidative stress biomarkers (MDA, SOD and CAT) among all groups, compared to the control group. Only the group III that received $1.76 \mathrm{mg} / \mathrm{kg}$ of AgNPs, showed a significant increase in CAT $(91.96 \pm 12.1, p<0.05)$ when compared to the control group $(68.34 \pm 10.6)$, Figure 4.
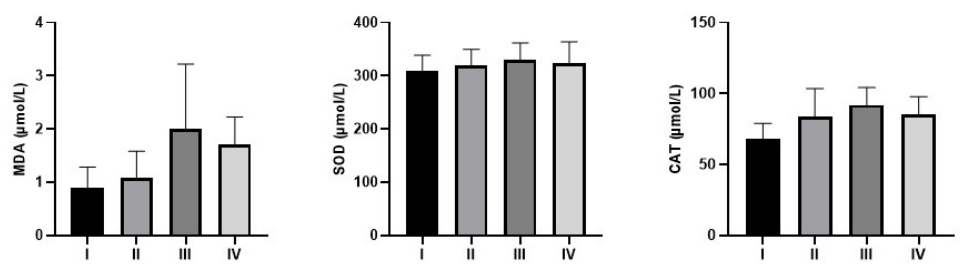

Figure 4 The effect of silver nanoparticles (AgNPs) administration on serum levels of:A)malondialdehyde (MDA),B)superoxide dismutase (SOD), and C) catalase (CAT) in rats. Data was presented as mean $\pm \mathrm{SD}(\mathrm{n}=6),{ }^{*}=p<0.05$. I, control group; II, group received $0.85 \mathrm{mg} / \mathrm{kg}$ AgNPs; III; group received1.76mg/kg AgNPs; IV, group received 3.53mg/kg AgNPs.

\section{Histopathology}

Histological examination of the kidneys and liver sections of the AgNPs administered groups showed no changes when compared with the control. Sections of kidney of the con- 
trol group rats showing normal glomeruli and normal renal cortical tubules as shown in Figure 5: A-D. Sections of liver of the control group revealed normal hepatocyte, normal portal vein (PV), central vein and sinusoid as presented in Figure 6: A-D.

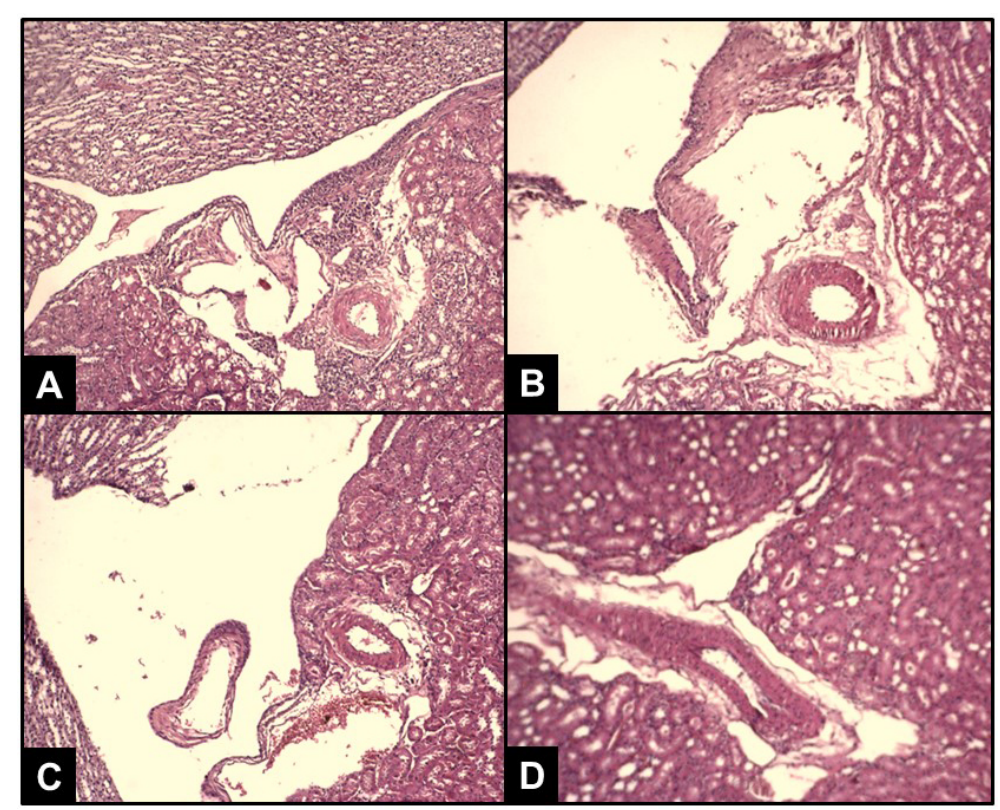

Figure 5 Photomicrograph of sections of kidney in:A)group I (control group), B)group II (group received $0.85 \mathrm{mg} / \mathrm{kg} \mathrm{AgNPs),} \mathrm{C)group} \mathrm{III} \mathrm{(group} \mathrm{received1.76mg/kg} \mathrm{AgNPs),} \mathrm{andD)} \mathrm{group} \mathrm{IV} \mathrm{(group}$ received 3.53mg/kg AgNPs); H\&E staining (10X).

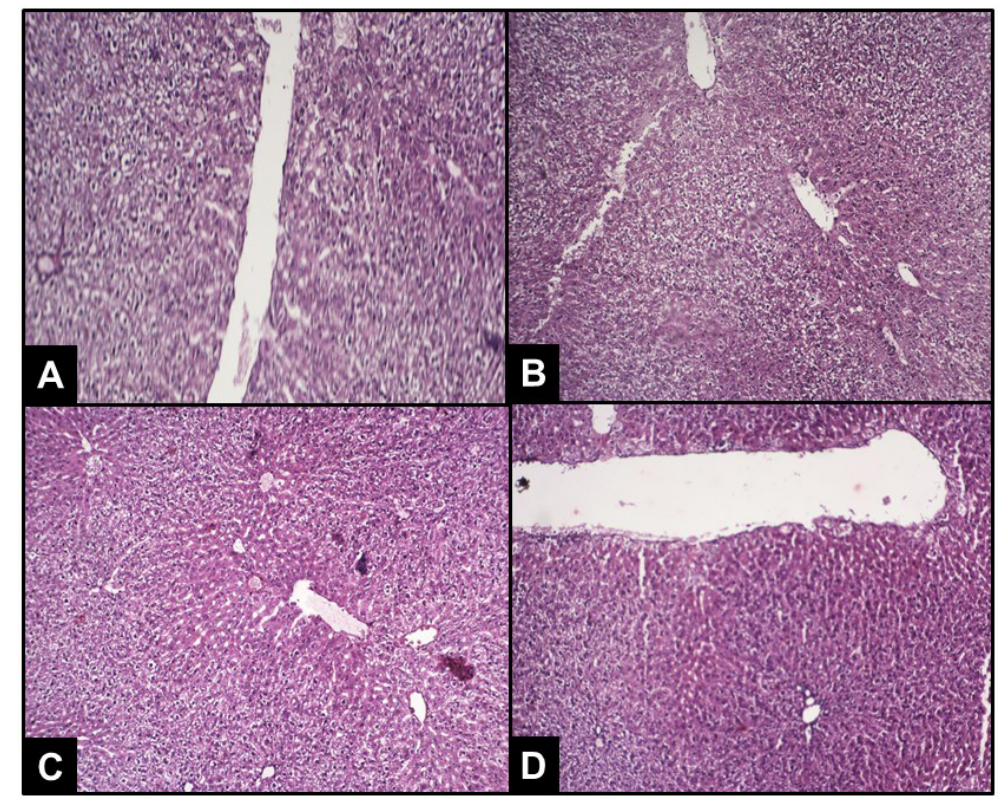

Figure 6 Photomicrograph of sections of liver in:A)group I (control group), B)group II (group received $0.85 \mathrm{mg} / \mathrm{kg} \mathrm{AgNPs}$ ), C) group III (group received1.76mg/kg AgNPs), andD) group IV (group received $3.53 \mathrm{mg} / \mathrm{kg}$ AgNPs); H\&E staining (10X). 


\section{DISCUSSION}

Sub-acute toxicity is a method to assess the safety and toxicological effects of substances upon multiple exposures. ${ }^{18}$ The current study is essentially designed to assess the safe dose of biosynthesized AgNPs of C. zeylanicum bark extract in experimental rats. In an ideal acute toxicity study, the harmful effect of the chemical should occur within 14 days of administration. ${ }^{19}$ In the current study, AgNPs were synthesized using a simple method (green synthesis). Initially, we used methanolic bark extract of C. zeylanicum extract as a reducing agent to convert silver from $\mathrm{Ag}^{+}$to $\mathrm{Ag}^{0}$. The reduction process occurs due to the presence of bioactive phytochemicals in the C. zeylanicum bark extract. ${ }^{20}$ The solution's color changed from light brown to dark brown after 72 hours, indicating the fabrication of the AgNPs. This observation was in line with the findings of Kumararaja et al. (2019). ${ }^{20}$ The reduction of silver ions into silver nanoparticles occurs during plant extract exposure and is followed by a color change also reported by Rajesh et al. (2009). ${ }^{21}$ AgNPs, in the current study, were spherical in shape, smooth edged, with nanoscale sizes as the SEM and AFM images showed. These particles were with little aggregations and below $100 \mathrm{~nm}$ in range diameters of $60-80 \mathrm{~nm}$. This aggregation took place due to the presence of cell components on the surface of nanoparticles and acts as capping agent. ${ }^{22}$ Similar finding was reported by Gauthami et al. (2015). ${ }^{10}$

There was no evidence of toxicity in rats given different doses $(0.85,1.76$, and $3.53 \mathrm{mg} / \mathrm{kg})$ of AgNPs of C. zeylanicum during the study period, like mortality, tremors, writhing, convulsion, analgesia, increased motor activity, cyanosis, or anesthesia. The toxicity of AgNPs exposure, according to Kim and Ryu (2013), ${ }^{23}$ could not be explained just by the presence of $\mathrm{Ag}^{+}$in the NPs' solution. As a result, the bioactive compound present in the methanol extract of C. zeylanicum act as a reducing agents for $\mathrm{Ag}^{+}$into $\mathrm{Ag}^{0},{ }^{10}$ making AgNPs safe for administration. Furthermore, this may be influenced by the size and dose of the NPs. The results of the body weight of the rat and body weight gain presented in this study showed that the AgNPs of C. zeylanicum did not cause any significant changes. The body weight of rats increased gradually in all of the groups investigated. This indicates that the AgNPs of $C$. zeylanicum does not have the ability to increase or encourage weight loss. Changes in body weight provide information about the effects of a substance administered. ${ }^{13}$ Liver and kidney are major target organs for several substances due to their critical role in various body processes including detoxification and excretion. ${ }^{24}$ In this respect, it's critical that studies examining the biochemical effects of substances take a close look at the status of these organs. Oxidative stress is one mechanism of injury to these organs, which can result in the generation of malondialdehyde (MDA) and 4-hydroxynonenal, as well as changes in antioxidant enzyme activity. ${ }^{25}$ In this study, the AgNPs did not cause any significant change in MDA, SOD and CAT levels when compared with the controls. Similar findings were achieved by Nasir et al. $(2015)^{26}$ who reported that no significant change occurs in oxidative stress biomarkers (MDA, CAT and SOD) after 14 days from AgNPs administration. As for indicators of how to assess renal dysfunction, measurements of urea and creatinine are used. The serum levels of creatinine and urea in all groups of rats who were given AgNPs 
with different doses $(0.85,1.76$ and $3.53 \mathrm{mg} / \mathrm{kg})$ were not significantly different from the control group. This implies that the AgNPs at these doses did not have any toxic effect on renal cells. In addition, this study has also been evaluated the serum levels of liver enzymes (ALT, AST). They were not significantly different in AgNPs-treated groups when compared to the controls and this was in agreement with findings by Nasir et al. (2015). ${ }^{26}$ In cytotoxic and cholestatic hepatic injury, some serum liver enzymes, including AST and ALT, have been observed to increase. ${ }^{27}$ Thus, the hepatic injury was not suspected based on our results. Also, no histopathological changes in the liver and kidney were noticed in treated groups when compared with the control group.

Still, the current study is subject to certain limitations. Firstly, the small number of rats used in this experiment. Secondly, the short duration of biosynthesized AgNPs administration. Thirdly, we weren't study the effect of toxicity of AgNPs on other organs, such as the heart and the brain. However, and in spite of these limitations, this is the first study about the toxicity of biosynthesized AgNPs (using bark extract of C. zeylanicum) on experimental rats.

\section{CONCLUSIONS}

The current study has proven the safety of AgNPs, which were tested on rats at various doses. No mortality, oxidative stress, hepatic and renal dysfunction, and no changes in liver and kidney histopathology were observed.

\section{ABBREVIATIONS}

AFM, Atomic force microscopy; AgNPs, silver nanoparticles; AST, alanine transaminase; AST, aspartate transaminase; CAT, catalase; GSH, glutathione; H\&E, hematoxylin and eosin; MDA, Malondialdehyde; SEM, Scanning electron microscopy; SOD, superoxide dismutase.

\section{ACKNOWLEDEGMENTS}

The authors are extremely thankful to the staff working in the Ministry of Science and Technology, Directorate of Environment and Water, especially Dr. Labeeb Ahmed and Dr. Suha Mohamed for their kind assistance in the preparation of silver nanoparticles and their characterization. The authors are also thanking to Mr. Hatem M. Hatem for his contribution to working at the animal house. 


\section{DECLARATIONS}

\section{Authors' contributions}

Conceptualization: SHA, HAA. Data curation: SHA. Formal analysis: SHA. Funding acquisition: N/A. Investigation: SHA. Methodology: SHA, MHA, HAA. Project administration: SHA. Resources: SHA. Software: SHA, HAA. Supervision: MHA. Validation: SHA. Visualization: SHA, MHA. Writing-original draft: SHA. Writing-review \& editing: MHA, HAA.

\section{Conflict of interest}

The authors declared that there is no conflict of interest.

\section{Ethical approvals}

The institutional review board and research ethics committee at the College of Veterinary Medicine, University of Basra (Basrah, Iraq) has approved this study (No.: 3/18 on 01/07/2019).

\section{Data availability}

The data that support the findings of this study are available from the corresponding author, upon reasonable request.

\section{Funding resources}

This work did not receive any fund.

\section{REFERENCES}

1. Sankar R, Maheswari R, Karthik S, Shivashangari KS, Ravikumar V. Anticancer activity of Ficus religiosa engineered copper oxide nanoparticles. Mater Sci Eng C. 2014;44:234-239. Available from: 10.1016/j.msec.2014.08.030.

2. Jasim NA, Al-Gasha’a FA, Al-Marjani MF, Al-Rahal AH, Abid HA, Al-Kadhmi NA, et al. ZnO nanoparticles inhibit growth and biofilm formation of vancomycinresistant S. aureus (VRSA). Biocatal Agric Biotechnol. 2020;29:101745-101745. Available from: 10.1016/j.bcab.2020.101745.

3. Yaseen SM, Abid HA, Kadhim AA, Aboglida EE. Antibacterial activity of palm heart extracts collected from Iraqi Phoenix dactylifera L. J Tech. 2020;1:52-59. Available from: 10.51173/jt.v1i1.70.

4. Dhillon GS, Brar SK, Kaur S, Verma M. Green approach for nanoparticle biosynthesis by fungi: current trends and applications. Crit Rev Biotechnol. 2012;32(1):49-73. Available from: 10.3109/07388551.2010.550568.

5. Sabri MA, Umer A, Awan GH, Hassan MF, Hasnain A. Selection of suitable biological method for the synthesis of silver nanoparticles. Nanomater Nanotechnol. 2016;6:29-29. Available from: 10.5772/62644. 
6. Oberdörster G. Safety assessment for nanotechnology and nanomedicine: concepts of nanotoxicology. J Intern Med. 2010;267(1):89-105. Available from: 10.1111/j. 1365-2796.2009.02187.x.

7. Shafey AME. Green synthesis of metal and metal oxide nanoparticles from plant leaf extracts and their applications: A review. Green Process Synth. 2020;9(1):304-339. Available from: 10.1515/gps-2020-0031.

8. Leela A, Vivekanandan M. Tapping the unexploited plant resources for the synthesis. Afr J Biotechnol. 2008;7:3162-3165.

9. Ansar S, Tabassum H, Aladwan NSM, Ali MN, Almaarik B, AlMahrouqi S, et al. Eco friendly silver nanoparticles synthesis by Brassica oleracea and its antibacterial, anticancer and antioxidant properties. Sci Rep. 2020;10(1):1-12. Available from: 10.1038/s41598-020-74371-8.

10. Gauthami M, Srinivasan N, Goud NM, Boopalan K, Thirumurugan K. Synthesis of silver nanoparticles using Cinnamomum zeylanicum bark extract and its antioxidant activity. Nanosci Nanotechnol - Asia. 2015;5(1):2-7. Available from: 10.2174/221068120501150728103209.

11. Kouame K, Peter AI, Akang EN, Adana M, Moodley R, Naidu EC, et al. Effect of longterm administration of Cinnamomum cassia silver nanoparticles on organs (kidneys and liver) of Sprague-Dawley rats. Turk J Biol. 2018;42(6):498-505. Available from: 10.3906/biy-1805-103.

12. Aamir K, Khan HU, Hossain CF. Oral toxicity of arjunolic acid on hematological, biochemical and histopathological investigations in female Sprague Dawley rats. PeerJ. 2019;2019:1-24. Available from: 10.7717/peerj.8045.

13. Ebohon O, Irabor F, Omoregie ES. Sub-acute toxicity study of methanol extract of Tetrorchidium didymostemon leaves using biochemical analyses and gene expression in Wistar rats. Heliyon. 2020;6(6):e04313-e04313. Available from: 10.1016/j. heliyon.2020.e04313.

14. Linares R, Rosas G, Vieyra E, Ramírez DA, Velázquez DR, Espinoza JA, et al. In adult rats with polycystic ovarian syndrome, unilateral or bilateral vagotomy modifies the noradrenergic concentration in the ovaries and the celiac superior mesenteric ganglia in different ways. Front Physiol. 2019;10:1-9. Available from: 10.3389/fphys.2019. 01309.

15. Yaseen SM, Abid HA, Al-Obaidi MAW. Disturbed levels of non-enzymatic antioxidants and malondialdehyde among makeup users. J Tech. 2020;2(1):42-48. Available from: 10.51173/jt.v2i1.166.

16. Lobo V, Patil A, Phatak A, Chandra N. Free radicals, antioxidants and functional foods: Impact on human health. Pharmacogn Rev. 2010;4(8):118-118. Available from: 10.4103/0973-7847.70902.

17. Swanson E, Wallace WD. Methods in Molecular Biology. In: Day CE, et al., editors. Histopathology Methods and Protocols. vol. 1180 of Methods in Molecular Biology. Humana Press, New York, NY; 2014. p. 283-291. Available from: 10.1007/978-14939-1050-2. 
18. Anderson JM, Biocompatibility. Biocompatibility. In: and others, editor. Polymer Science: A Comprehensive Reference. vol. 10. Elsevier; 2012. p. 363-383. Available from: 10.1016/B978-0-444-53349-4.00229-6.

19. Bhardwaj S, Gupta D. Study of acute, sub acute and chronic toxicity test. Int J Curr Pharm Res. 2012;2:103-129.

20. Kumararaja G, Sundaraganapathy R, Constantine I. Comparative studies on synthesized silver nanoparticles using Artemisia vulgaris Linn., and Cinnamomum zeylanicum Nees., for their antifungal activity. J Pharm Sci Res. 2019;11:2558-2565.

21. Rajesh RW, Jaya LR, Niranjan KS. Phytosynthesis of silver nanoparticle using Gliricidia sepium (Jacq.). Curr Nanosci. 2009;5:117-122. Available from: 10.2174/ 157341309787314674.

22. Bélteky P, Rónavári A, Igaz N, Szerencsés B, Tóth IY, Pfeiffer I, et al. Silver nanoparticles: aggregation behavior in biorelevant conditions and its impact on biological activity. Int J Nanomed. 2019; Volume 14:667-687. Available from: 10.2147/ijn. s185965.

23. Kim S, Ryu DY. Silver nanoparticle-induced oxidative stress, genotoxicity and apoptosis in cultured cells and animal tissues. J Appl Toxicol. 2013;33:78-89. Available from: 10.1002/jat.2792.

24. Bello I, Bakkouri A, Tabana Y, Al-Hindi B, Al-Mansoub M, Mahmud R, et al. Acute and sub-acute toxicity evaluation of the methanolic extract of Alstonia scholaris stem bark. Med Sci. 2016;4(1):4-4. Available from: 10.3390/medsci4010004.

25. Ayala A, Muñoz MF, Argüelles S. Lipid peroxidation: Production, metabolism, and signaling mechanisms of malondialdehyde and 4-hydroxy-2-nonenal. Oxid Med Cell Longev. 2014;2014:1-31. Available from: 10.1155/2014/360438.

26. Nasir GA, Samir HF, Mohammed AK. Effect of silver nanoparticals in liver function enzymes and oxidative stress levels. World J Pharm Res. 2015;4:2080-2089.

27. Zimmerman HJ. Drug-Induced Liver Disease. Drugs. 1978;16:25-45. Available from: 10.2165/00003495-197816010-00002. 